\title{
Evaluation of Graded Transcutaneous Levator Muscle Recession with Mullerectomy for Treatment of Upper Eyelid Retraction in Dysthyroid Patients
}

\author{
Ahmad H. and Abd Elmagid M. Tag Eldin \\ Ophthalmic Department, Faculty of Medicine, Al-Azhar University
}

\begin{abstract}
Purpose: To evaluate the efficacy, advantages and disadvantages of graded transcutaneous levator muscle recession with mullerectomy for treatment of dysthyroid upper eyelid retraction.

Design: Randomized, prospective and interventional case series.

Methods: This prospective interventional case series comprised 20 eyes of ten patients. Inclusion criteria included patients with bilateral upper eyelid retraction, with or without other manifestations of thyroid orbitopathy, without diplopia and with stable thyroid state for at least 6 months. All patients were subjected to graded transcutaneous levator muscle recession with mullerectomy after full ophthalmological assessment. All patients included in the study have signed a written consent and the study has been approved by the ethical committee of AL-AZHAR faculty of medicine.

Results: Postoperatively the mean decrease of MRD1 was $2.87 \mathrm{~mm}$. Mean improvement of lagophthalmos was 0.78 $\mathrm{mm}$. Mean increase of upper lid crease height was $0.65 \mathrm{~mm}$. Good lid contour achieved in $85.9 \%$. Temporal flare persisted in one lid (5\%) and nasal ptosis was reported in two eye lids (10\%).

Conclusion: According to this study graded transcutaneous mullerectomy with levator muscle recession is a good and a reliable surgical technique for correction of dysthyroid upper lid retraction and is successful as a lengthening procedure for retracted upper eyelid regardless the severity of retraction with significant symptomatic and cosmetic improvement.
\end{abstract}

Keywords: Mullerectomy, Upper eyelid retraction, Dysthyroid, Graded transcutaneous levator muscle recession, Levator lengthening.

\section{INTRODUCTION}

Lid retraction is a disorder of eyelid malposition characterized by appearance of a band of white sclera between the limbus and the eyelid margin, when the eye in the primary position ${ }^{[1]}$.

Eyelid retraction is the most common ophthalmic feature of thyroid-related orbitopathy, occurring unilaterally or bilaterally in more than $90 \%$ of patients at some point in their clinical course ${ }^{[2]}$. The exact cause of lid retraction in Graves ophthalmopathy is not completely understood, but several factors seems to be contributory. In upper lid these factors include: muscle contraction due to adrenergic stimulation; involvement of Mullerlevator complex by Graves disease process; overaction of levator-superior rectus complex in response to hypophoria produced by inferior rectus fibrosis; and adhesion of the levator to the orbital septum and neighboring subcutaneous tissues. In the lower lid: stimulation of sympathetic muscle plays a role; fibrosis of inferior rectus exerts a retracting action; proptosis if present plays a role; and adhesion of inferior retractors to neighboring subcutaneous tissues ${ }^{[3,4,5]}$.

Retraction of the upper eyelid may cause decreased excursion of the eyelid and lagophthalmos, which may result in exposure keratopathy. The effect of these conditions resulting from eyelid involvement range from minor ocular irritation and discomfort to vision-threatening corneal decompensation, potentially resulting in severe vision loss. Exposure keratopathy may lead to foreign body sensation, discomfort, epiphora, keratitis, formation of erosive corneal ulcers, burning sensation, reactive epiphora, photophobia, blurred vision, conjunctival irritation, and decreased visual acuity due to the chronic exposure of the cornea and conjunctiva and cosmetic concerns with the related emotional stress. In thyroid-related orbitopathy, upper eyelid retraction present in primary gaze is called Dalrymple sign, and an abnormally high upper eyelid in down gaze is called the Von Graefe sign or lid lag ${ }^{[6]}$.

The surgical treatment of eyelid retraction presents challenging management problems to the surgeon. Although patients frequently attained postoperative reduction in their eyelid retraction with many prior techniques, occasional contour defects and the occasional residual lid retraction more prominent temporally led to the search for new or modified techniques for the correction of upper eyelid retraction. The large number of surgical procedures described for the correction of upper eyelid retraction attests to the difficulty of reliably correcting this disease process. In thyroid-related orbitopathy, results are often unpredictable with inconsistent eyelid position and contour abnormalities. Recurrent retraction, overcorrection, and undercorrection are recognized complications of eyelid retraction surgery secondary to thyroid-related orbitopathy. Several operations may be necessary to achieve complete success ${ }^{[7]}$.

Muller's muscle surgery alone corrects approximately $2 \mathrm{~mm}$ of upper lid retraction, while 
Muller's muscle and levator surgery corrects approximately 4-6 $\mathrm{mm}$ of upper lid retraction ${ }^{[8]}$.

The aim of this study is to evaluate the efficacy, advantages, and disadvantages of graded transcutaneous levator muscle recession with Mullerectomy for treatment of dysthyroid upper eyelid retraction.

\section{Patients and Methods}

This prospective interventional case series comprised 20 eyes of ten patients. Inclusion criteria included patients with bilateral upper eyelid retraction, with or without other manifestations of thyroid orbitopathy, without diplopia and with stable thyroid state for at least 6 months. Exclusion criteria included clinically or biochemically unstable dysthyroid disease, proptosis more than $22 \mathrm{~mm}$ or diplopia due to restrictive myopathy.

All patients were subjected to full clinical ophthalmological examination including: best corrected visual acuity, ocular motility, slit lamp examination, intraocular pressure measurement and fundus examination. The study was approved by the Ethics Board of Al-Azhar University.

Upper eyelid examination including: Amount of lid retraction using Margin Reflex Distance (MRD), which is the distance between corneal light reflex and upper eyelid margin using a ruler, upper eyelid contour for temporal flare, upper eyelid crease height, vertical palpebral fissure height.

Investigations: Visual field and orbital CT or MRI. Systemic assessment of thyroid state was done in cooperation with an endocrinologist. Photography: external digital camera photos.

\section{Surgical technique:}

\section{Levator recession with mullerectomy}

Sterilization of operative field (upper lids, lower lids, eyebrows, forehead and nose of both sides) with Betadine (povidone - Iodine 5\%) solution and draping, $2 \%$ lidocaine with $0.5 \%$ bubivacaine is injected subcutaneously below the skin crease. A 4-0 silk traction suture is placed in the eyelid margin. The skin crease is incised, then orbicularis oculi muscle is dissected, then the orbital septum is incised and the pre apponeurotic fat is retracted to expose the levator apponeurosis. The apponeurosis is dissected from upper border of tarsus and underlying Muller's muscle starting temporally with extirpation of the lateral horn in most cases to alleviate temporal flare. Dissection then extends medially according to the preoperative lid contour. Injection of saline under the apponeurosis may make dissection easier. Muller`s muscle is then identified by vertical direction of its fibers and presence of vascular arcade at its lower border. Muller`s muscle is dissected from underlying conjunctiva from below to above then excised as far from its lower border as possible. The levator apponeurosis is then sutured with 6-0 Vicryl to the underlying conjunctiva. The suture distance is adjusted from the upper border of tarsus according to the degree of retraction, and this can be measured by asking the patient to sit up and assessing lid height and contour (Figure3). Then orbicularis oculi muscle and skin are sutured in separate layers. Skin is closed with a running 5-0 Vicryl suture. A steroid antibiotic ointment is then applied.

Follow up was carried out at one week, one month, and 3 months, after surgery. It included evaluation of MRD1, improvement of lagophthalmos, and mean increase of upper lid crease height.

\section{RESULTS}

In this study, the mean age + SD was $40.54 \pm$ 10.34 years. Seven females $(70 \%)$ and 3 males $(30 \%)$ were included. Duration of thyroid eye disease (TED) $\pm \mathrm{SD}$ was $1.89 \pm 0.80$ years. Mean preoperative Hertler exophthalmometry \pm SD was $20.01 \pm$ $0.96 \mathrm{~mm}$. Mean MRD \pm SD was $7.03 \pm 0.75 \mathrm{~mm}$. Mean palpebral vertical fissure height \pm SD was $12.20 \mathrm{~mm} \pm 0.48 \mathrm{~mm}$. Mean upper eyelid crease height \pm SD was $9.54 \pm 0.64 \mathrm{~mm}$. Temporal flare was present in 12 lids (60\%).

Table (1): Clinical data of the studied patients.

\begin{tabular}{|c|c|c|c|c|c|c|c|c|}
\hline \multirow{2}{*}{$\begin{array}{l}\text { Case } \\
\text { No. }\end{array}$} & \multirow{2}{*}{ Sex } & \multirow{2}{*}{$\begin{array}{c}\text { Age } \\
\text { [years] }\end{array}$} & \multicolumn{2}{|c|}{$\operatorname{MRD}(\mathbf{m m})$} & \multicolumn{2}{|c|}{$\begin{array}{l}\text { Palpebral vertical } \\
\text { fissure height ( } \mathrm{mm})\end{array}$} & \multicolumn{2}{|c|}{$\begin{array}{c}\text { Eyelid crease } \\
\text { height }(\mathrm{mm})\end{array}$} \\
\hline & & & RT & LT & RT & LT & RT & LT \\
\hline 1 & 오 & 35 & 7.00 & 7.00 & 12.25 & 12.20 & 9.54 & 9.54 \\
\hline 2 & o & 36 & 7.53 & 7.55 & 11.80 & 12.00 & 9.65 & 9.50 \\
\hline 3 & 오 & 47 & 7.45 & 7.30 & 13.00 & 12.10 & 11.00 & 11.00 \\
\hline 4 & $\hat{0}$ & 40 & 6.95 & 7.00 & 12.20 & 12.15 & 9.50 & 9.45 \\
\hline 5 & 우 & 41 & 7.50 & 7.00 & 12.30 & 11.40 & 9.20 & 9.50 \\
\hline 6 & 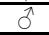 & 41 & 6.80 & 6.85 & 11.10 & 11.05 & 9.50 & 9.55 \\
\hline 7 & 우 & 35 & 7.00 & 7.40 & 12.15 & 12.20 & 9.10 & 9.40 \\
\hline 8 & q & 44 & 7.35 & 7.25 & 12.25 & 12.25 & 9.45 & 9.50 \\
\hline 9 & 운 & 38 & 6.80 & 7.00 & 12.90 & 13.10 & 9.30 & 9.20 \\
\hline 10 & q & 46 & 6.75 & 8.40 & 11.05 & 12.00 & 9.00 & 9.50 \\
\hline
\end{tabular}

Postoperatively, the mean decrease of MRD was $2.77 \mathrm{~mm}$. Mean improvement of palpebral vertical fissure height was $0.75 \mathrm{~mm}$. Mean increase of upper lid crease height was $0.6 \mathrm{~mm}$. Good lid contour achieved in $86.7 \%$. Temporal flare persisted in one lid $(5 \%)$ and nasal ptosis was reported in two lids $(10 \%)$.

Table (2): Preoperative and postoperative data.

\begin{tabular}{|l|l|c|c|c|}
\hline \multicolumn{2}{|l|}{} & Preoperative & Postoperative & P-value \\
\hline MRD(mm) & Mean \pm SD & $\begin{array}{c}7.07 \pm 0.78 \\
6.75-8.4\end{array}$ & $\begin{array}{c}4.70 \pm 0.78 \\
3.50-5.3\end{array}$ & 0.001 \\
\hline Ralpebral vertical & Rean \pm SD & $12.20 \pm 0.48$ & $8.50 \pm 0.75$ & \multirow{2}{*}{0.005} \\
fissure height (mm) & Range & $1-2$ & $0.5-0.5$ & \\
\hline \multirow{2}{*}{ Upper lid crease (mm) } & Mean \pm SD & $\begin{array}{c}9.47 \pm 0.61 \\
9-11\end{array}$ & $\begin{array}{c}10.0 \pm 0.46 \\
9-105\end{array}$ & 0.005 \\
\hline
\end{tabular}

Results proved statistically significant changes of lids operated on using mullerectomy with levator recession as lid lengthening surgery. There was a statistically significant decrease of MRD ( $P$ $=0.001)$, statistically significant improvement of vertical palpebral fissure height $(\mathrm{P}=0.005)$, and statistically significant increase of lid crease height $(\mathrm{P}$ $=0.005$ ).

Ecchymosis and heamatoma of lid during local infiltration anesthesia was reported in two lids. 
Skin burn was reported once during cauterization. Severe bleeding was reported twice during Muller's muscle dissection. Improper dissection of Muller's muscle as one layer and excision in pieces was reported three times. Conjunctival button holing was reported once. Persistent temporal flare occurred in two cases. Nasal ptosis was reported in one case.

\section{DISCUSSION}

Many surgical techniques have been developed to address eyelid retraction with varying results. Identifying and evaluating the anatomical and pathophysiological factors involved will assist in its surgical treatment. This prospective study evaluated the graded levator recession with Müllerectomy for treating upper eyelid retraction in dysthyroid patients. Blaskovic severed the levator and, presumably, Müller's muscle from the superior tarsal border and retracted them with sutures ${ }^{[9]}$.

In 1972, Putterman and Urist' described a procedure for excising Müller's muscle with or without recession of the levator aponeurosis. The patient is awake during the procedure and is sitting up at various times either to match the level of the affected lid to the unaffected lid in unilateral cases or to achieve a functionally and cosmetically acceptable level in bilateral cases ${ }^{[10]}$. Baylis et al described the recession of the levator aponeurosis and Müller's muscles twice the distance of the measured retraction and suturing them to conjunctiva $^{[11]}$.

The excision of Muller's muscle and levator recession in the manner described is an effective procedure for treating thyroid-related retraction of the upper eyelid as it is based on the theories of the physiologic (excision of Muller's muscle which is presumed to has sympathetic overstimulation) and anatomic origin of the condition (recession of levator apponeurosis which is presumed to be shortened by fibrosis) ${ }^{[12]}$.

Daniel (2007) reported that it is a graded procedure and patient is sitting up during the procedure so we can adjust the amount of levator recession until the desired level and contour are achieved. Overcorrection can be recognized during surgery and dealt with by reattaching the recessed tissues to the underlying conjunctiva at a lower level ${ }^{[13]}$.

The approach was chosen in this study was trascutaneously not transcojunctivally for many reasons: Trascutaneous approach allows good exposure of lid retractors specially horns of levator that is a mandatory step to correct temporal flare, good heamostasis during Muller`s muscle dissection and excision which is a bloody step due to vascular arcade overlying the muscle, surgical steps by far simulate ptosis surgery and upside down anatomy of transcojunctival approach makes it somewhat confusing .

In our study, the mean decrease of MRD1was $2.87 \mathrm{~mm}$, mean improvement of lagophthalmos was
$0.78 \mathrm{~mm}$ and mean increase of upper lid crease height was $0.65 \mathrm{~mm}$. Good lid contour was achieved in $85.9 \%$. Temporal flare persisted in one lid (5\%), and nasal ptosis was reported in two eye lids (10\%).

Results proved statistically significant changes of lids operated on regarding decrease of MRD1 (P $=0.000)$, improvement of lagophthalmos and increase of lid crease height $(\mathrm{P}=0.005)$.

Harvey and Anderson did transcutaneous mullerectomy with levator recession in 20 eyelids with dysthyroid upper eyelid retraction (14 patients). They considered that the results are "good" if the contour is good and the postoperative lid height is within $1 \mathrm{~mm}$ of either the desired height (in bilateral surgery) or the other lid (in unilateral surgery). Results are considered "satisfactory" if the lid height varied greater than $1 \mathrm{~mm}$ of the desired height, yet cosmetically and functionally are acceptable and do not require reoperation. "Poor" results are those who require reoperation for excessive over - or undercorrection of contour abnormalities.

In their study, fifteen of these lids were considered good (75\%), and two were considered as satisfactory results after one operation. Three lids were classed as poor. Of these three, two were overcorrections, which were operated on again, and then were considered good or satisfactory. Almost all of overcorrections occurred in their first few cases due to aiming at overcorrection at the time of surgery expecting some postoperative recurrence of retraction. It rapidly became evident that the lids did not rise postoperatively ${ }^{[14]}$.

Nixon et al did modified transcutaneous Mullerectomy with levator recession dissecting only lateral two thirds of apponurosis in 22 eyelids with dysthyroid upper eyelid retraction (12 patients), 19 of them had good results ${ }^{[15]}$

In this study, only two cases (lids) of nasal ptosis were reported which may be due to early cutting of medial horn of levator muscle and recession of nasal part of the muscle. So preservation of medial horn is preferred and extirpation of the apponeurosis should include lateral part then proceeds medially according to the pattern of lid retraction.

There are also two cases with severe lid retraction and marked temporal flare showing persistent temporal flare in spite of good extirpation of the lateral horn, complete excision of Muller's muscle, exposure of underlying conjunctiva and with marked temporal recession of the levator aponeurosis.

Eyelid contour and symmetry of the eyelid crease and fold are other criteria that are important in assessing postoperative results in the surgical correction of eyelid retraction. Postoperatively, patients may have an excellent lid level, but the contour, temporal flare, lid fold, and crease variations may produce an aesthetically unacceptable result in that the lid still looks quite abnormal. In reviewing the postoperative results, the eyelid crease and contour results, which were not 
quantified in this study, were compared to the preoperative status. These results are highly subjective, but all patients were pleased with their final result.

Many aspects of these procedures are similar to other operations used for the treatment of eyelid retraction. The success rates for treatment of eyelids have been reported in the range of $20 \%$ to $50 \%$, and the use of adjustable techniques and early postoperative adjustment increases the success rate up to $84 \%$ of cases [16-18]

\section{CONCLUSION}

According to this study graded transcutaneous levator muscle recession with mullerectomy is a good and a reliable surgical technique for correction of dysthyroid upper lid retraction and successful as a lengthening procedures for retracted upper eyelid regardless severity of retraction with significant symptomatic and cosmetic improvement.

\section{REFERENCES}

1. Levine M, Chu A(1991): Surgical treatment of thyroid related lid retraction. Ophthalmic surgery , 22: 2-11.

2. Bartley GB(1994): The epidemiologic characteristics and clinical course of ophthalmopathy associated with autoimmune thyroid disease in Olmsted County, Minnesota. Trans Am Ophthalmol Soc .,92:477-588.

3. Fruch B, Grill R, Musch D(1984): Lid protractor force generation in Graves' eye disease. Ophthalmology,93:8-13.

4. Feldon SE, Levin L(1990): Graves' ophthalmology, aetiology of upper eyelid retraction in Graves' ophthalmopathy. $\mathrm{Br} \mathrm{J}$ Ophthalmol.,74:484-485.

5. Clauser L, Galie M, Sarti E(2001): Rationale of treatment in Graves's ophthalmopathy. Plast Reconstr Surg .,108:1880-1894.

6. Cockerham KP, Hidayat AA, Brown HG (2002): Clinicopathologic evaluation of the Muller muscle in thyroid-associated orbitopathy. Ophthal Plast Reconstr Surg.,18:11-17.
7. Chang EL, Rubin PAD (2002): Upper and lower eyelid retraction. Int Ophthalmol Clin., 42:45-59.

8. Putterman A (1981): Surgical treatment of thyroid-related upper eyelid retraction, Graded Muller muscle excision and levator recession. Opthalmology, 88:499-507.

9. Blaskovic L, Berens C, King J (1961): Atlas of Ophthalmic Surgery. Philadelphia, JB Lippincott Co., p 76.

10. Putterman A, Urist M(1974): Surgical anatomy of the orbital septum. Ann Ophthalmol., 6:290-298.

11. Baylis H, Cies W, Kamin O(1976): Correction of upper eyelid retraction. Am J Ophthalmol.,82:790-794.

12. Karesh J(2000): Eyelid Retraction : Evaluation ,Management and treatment. In J A Mauriello(Ed). Unfavorable Results of Eyelid and lacrimal surgery. Prevention and Management. Butterworth -Heinemann, p 275.

13. Daniel P.S(2007): The graded levator hinge procedure for correction of upper eye lid retraction .Trans Am Ophthalmol Soc.,105: 481-512.

14. Harvey J, Anderson R(1981): The Aponeurotic Approach to eyelid Retraction. Ophthalmology, 88:513-524.

15. Nixon D, Corin S, , Veloudios A,Harvey J(1991):Modifed levator aponeurosis recession for upper eyelid retraction in Graves' disease. Ophthalmic Surg.,22:313-317.

16. Bahn R(2010): Graves' ophthalmopathy. N Engl J Med., 362(8):726-738.

17. Bahn R, Iyer S(2012): "Immunopathogenesis of Graves' ophthalmopathy: the role of the TSH receptor," Best Practice and Research. Clinical Endocrinology and Metabolism, 26: 281-289.

18. Banga P, Nielsen H, Gilbert J, El Fassi D, Hegedus L(2008): Application of new therapies in Graves' disease and thyroidassociated ophthalmopathy: animal models and translation to human clinical trials. Thyroid, 18: 973-981. 